\title{
Fault Modeling and Testing for Analog Circuits in Complex Space Based on Supply Current and Output Voltage
}

\author{
Hongzhi Hu, ${ }^{1,2}$ Shulin Tian, ${ }^{1}$ and Qing Guo ${ }^{2}$ \\ ${ }^{1}$ School of Automation Engineering, University of Electronic Science and Technology of China, Chengdu 611731, China \\ ${ }^{2}$ Guangxi Key Laboratory of Automatic Detecting Technology and Instrument, Guilin University of Electronic Technology, \\ Guilin 541004, China
}

Correspondence should be addressed to Hongzhi Hu; huhz@guet.edu.cn

Received 14 October 2014; Revised 22 February 2015; Accepted 23 February 2015

Academic Editor: Hossein Torkaman

Copyright (C) 2015 Hongzhi Hu et al. This is an open access article distributed under the Creative Commons Attribution License, which permits unrestricted use, distribution, and reproduction in any medium, provided the original work is properly cited.

\begin{abstract}
This paper deals with the modeling of fault for analog circuits. A two-dimensional (2D) fault model is first proposed based on collaborative analysis of supply current and output voltage. This model is a family of circle loci on the complex plane, and it simplifies greatly the algorithms for test point selection and potential fault simulations, which are primary difficulties in fault diagnosis of analog circuits. Furthermore, in order to reduce the difficulty of fault location, an improved fault model in threedimensional (3D) complex space is proposed, which achieves a far better fault detection ratio (FDR) against measurement error and parametric tolerance. To address the problem of fault masking in both $2 \mathrm{D}$ and $3 \mathrm{D}$ fault models, this paper proposes an effective design for testability (DFT) method. By adding redundant bypassing-components in the circuit under test (CUT), this method achieves excellent fault isolation ratio (FIR) in ambiguity group isolation. The efficacy of the proposed model and testing method is validated through experimental results provided in this paper.
\end{abstract}

\section{Introduction}

Over the past two decades, fault detection and diagnosis of analog circuits become an important research area where a number of corresponding theories and techniques have been developed. Among the researches in this area, fault dictionary is one of the most important methods that have attracted great interests [1-5]. Various circuit variables (e.g., voltage, current, and frequency) have been used in fault dictionary to obtain fault signatures [6-8]. In addition to the commonly employed measurement of output voltage, supply current testing has been applied widely in analog and mixed signal integrated circuits (ICs) with excellent fault coverage, especially for the catastrophic fault of CMOS ICs [9-11].

Despite the advantages of fault dictionary, there are still persistent challenges, such as test point selection and potential faults simulations, in applications of fault dictionary method for fault diagnosis of analog circuits. Wang and Yang proposed a slope fault mode, which not only reduces the required number of test points to two but also simplifies the potential fault simulation greatly [2]. However, the slope fault model is only limited to dynamic circuits. To achieve detection of parametric faults for analog circuits, Yang et al. proposes a complex-circle based fault model [3]. Although this proposed fault model is improved sequentially by optimal testing frequency selection [12] and fault location [13], the weakness of fault masking still remains. Moreover, a 3D model based on transfer function was proposed in [14]; it extends the distances between fault loci and improves the practicability for applications. Recently, Ma and Wang achieved detecting catastrophic faults and parametric faults, by analyzing the harmonic spectrum of output voltage and supply current [11]. Following the ideas of these researches, this paper proposes two improved fault models with satisfactory FDR based on collaborative analysis of output voltage and supply current. Moreover, to isolate ambiguity groups, a design for testability (DFT) method is proposed to achieve better fault isolation ratio (FIR) against the influence of analog tolerance and measurement errors.

This paper is organized as follows. First, the theory of collaborative fault model on 2D complex plane is introduced in Section 2. An example of a Sallen-Key filter is also provided 
to illustrate the theory in this section. In order to reduce the difficulty of fault location and isolation, an improved fault model in 3D complex space is proposed in Section 3. Furthermore, to deal with the ambiguity groups in the fault model, an innovative method of DFT is demonstrated in Section 4 to improve the FIR. Validation of the proposed method is provided by PSPICE. Finally, conclusions and future work are summarized in Section 5.

\section{Collaborative Fault Model on 2D Complex Plane}

2.1. Theorem of the 2D Collaborative Fault Model. A linear time-invariant passive CUT $N$ is assumed to contain $n$ components $F_{i}(1 \leq i \leq n) . N$ is assumed to be stimulated by a direct current (DC) power source $U_{d d}$ and a family of alternating current (AC) signals $U_{s k}(1 \leq k \leq m)$, where $m$ is the number of AC signals. Then a $(m+1)$-dimension vector $\mathbf{U}_{\mathbf{S}}$ is composed as follows:

$$
\mathbf{U}_{\mathbf{S}}=\left(\begin{array}{lllll}
U_{d d} & U_{s 1} & U_{s 2} & \cdots & U_{s m}
\end{array}\right)
$$

Furthermore, a single component $P$ is assumed to be the potential failure component in $N$, where

$$
P \in\left\{F_{i} \mid 1 \leq i \leq n\right\} .
$$

The voltage across $P$ is defined as $u_{F}$. According to the Substitution Theorem, a voltage source $u_{F}$ can be used to replace the component $P$ in the CUT. Therefore, both voltage $u_{F}$ and vector $\mathbf{U}_{\mathbf{S}}$ are regarded as the excitation of the CUT. Then the collaborative output $F_{c}(\cdot)$ is defined as follows:

$$
F_{c}(s, P)=\left(\begin{array}{ll}
U_{o} & I_{d d}
\end{array}\right)\left(\begin{array}{l}
c_{0} \\
c_{1}
\end{array}\right)
$$

where $U_{o}$ is the output voltage of CUT, $I_{d d}$ is the power supply current, and $s$ is the Laplacian operator. $c_{0}$ and $c_{1}$ are predefined coefficients. Furthermore, the corresponding transfer function matrices $\mathbf{H}$ and $\mathbf{H}_{\mathbf{F}}$ are defined as

$$
\begin{aligned}
\mathbf{H} & =\left(\begin{array}{llll}
H_{1 d d}(s) & H_{11}(s) & \cdots & H_{1 m}(s) \\
H_{2 d d}(s) & H_{21}(s) & \cdots & H_{2 m}(s)
\end{array}\right)^{T}, \\
\mathbf{H}_{\mathbf{F}} & =\left(\begin{array}{lll}
H_{1 F}(s) & \left.H_{2 F}(s)\right)
\end{array}\right.
\end{aligned}
$$

where $H_{1 d d}(s), H_{2 d d}(s), H_{1 k}(s)(1 \leq k \leq m), H_{2 k}(s)(1 \leq k \leq$ $m), H_{1 F}(s)$, and $H_{2 F}(s)$ are transfer functions with respect to $U_{o}$ and $I_{d d}$. Then, $F_{c}(\cdot)$ can be expressed as

$$
F_{c}(s, P)=\left(\mathbf{U}_{\mathbf{S}} \mathbf{H}+u_{F} \mathbf{H}_{\mathbf{F}}\right)\left(\begin{array}{c}
c_{0} \\
c_{1}
\end{array}\right) \text {. }
$$

In addition, according to the theories of circuit analysis, $u_{F}$ can be further defined as

$$
u_{F}=u_{\mathrm{oc}} \frac{Z_{F}}{Z_{0}+Z_{F}}
$$

where $u_{\mathrm{oc}}$ is open circuit voltage for the failure component $P$; $Z_{0}$ is corresponding Thevenin equivalent impedance, while $Z_{F}$ is the impedance of $P$. According to the Thevenin's Theorem, $u_{\mathrm{oc}}$ and $Z_{0}$ are independent of $Z_{F}$. Therefore,

$$
F_{c}(s, P)=\mathbf{U}_{\mathbf{S}} \mathbf{H}\left(\begin{array}{l}
c_{0} \\
c_{1}
\end{array}\right)+u_{\mathrm{oc}} \mathbf{H}_{\mathbf{F}}\left(\begin{array}{c}
c_{0} \\
c_{1}
\end{array}\right) \frac{Z_{F}}{Z_{0}+Z_{F}} .
$$

For any determinate frequency $\omega$, the Laplacian operator $s=j \omega$ is also an imaginary constant, where $j$ is imaginary unit vector. In addition, without loss of generality, $Z_{F}$ can be assumed to be a pure resistance $R_{F}$ or reactance $X_{F}$. Therefore, taking $R_{F}$ as an example,

$$
F_{c}(P)=\mathbf{U}_{\mathbf{S}} \mathbf{H}\left(\begin{array}{l}
c_{0} \\
c_{1}
\end{array}\right)+u_{\mathrm{oc}} \mathbf{H}_{\mathbf{F}}\left(\begin{array}{l}
c_{0} \\
c_{1}
\end{array}\right) \frac{R_{F}}{Z_{0}+R_{F}} .
$$

With the following assumptions,

$$
\begin{gathered}
\mathbf{U}_{\mathbf{S}} \mathbf{H}\left(\begin{array}{c}
c_{0} \\
c_{1}
\end{array}\right)=a_{1}+j b_{1}, \\
u_{\mathrm{oc}} \mathbf{H}_{\mathbf{F}}\left(\begin{array}{c}
c_{0} \\
c_{1}
\end{array}\right)=a_{2}+j b_{2}, \\
Z_{0}=R_{0}+j X_{0},
\end{gathered}
$$

where $a_{1}, b_{1}, a_{2}, b_{2}, R_{0}$, and $X_{0}$ are all real numbers, then

$$
\begin{array}{r}
F_{c}(P)=\left(a_{1}+j b_{1}\right)+\left(a_{2}+j b_{2}\right) \frac{R_{F}}{\left(R_{0}+j X_{0}\right)+R_{F}}, \\
\frac{R_{0}+j X_{0}}{R_{F}}=\frac{\left[a_{2}\left(F_{R}-a_{1}\right)+b_{2}\left(F_{I}-b_{1}\right)\right]}{\left(F_{R}-a_{1}\right)^{2}+\left(F_{I}-b_{1}\right)^{2}}-1 \\
+j \frac{\left[-a_{2}\left(F_{I}-b_{1}\right)+b_{2}\left(F_{R}-a_{1}\right)\right]}{\left(F_{R}-a_{1}\right)^{2}+\left(F_{I}-b_{1}\right)^{2}},
\end{array}
$$

where

$$
\begin{aligned}
& F_{R}=\operatorname{Re}\left[F_{c}(P)\right], \\
& F_{I}=\operatorname{Im}\left[F_{c}(P)\right] .
\end{aligned}
$$

If $R_{0}$ and $X_{0}$ are all equal to zero, the CUT is equal to an ideal voltage source $u_{\mathrm{oc}}$ corresponding to the component $P$. This means that variation in $Z_{F}$ will not affect $u_{F}$ and $F_{c}(P)$. Thus, we have

$$
\begin{gathered}
\frac{R_{0}}{R_{F}}=\frac{\left[a_{2}\left(F_{R}-a_{1}\right)+b_{2}\left(F_{I}-b_{1}\right)\right]}{\left(F_{R}-a_{1}\right)^{2}+\left(F_{I}-b_{1}\right)^{2}}-1, \\
\frac{X_{0}}{R_{F}}=\frac{\left[-a_{2}\left(F_{I}-b_{1}\right)+b_{2}\left(F_{R}-a_{1}\right)\right]}{\left(F_{R}-a_{1}\right)^{2}+\left(F_{I}-b_{1}\right)^{2}} .
\end{gathered}
$$

Then

$$
\frac{R_{0}}{X_{0}}=\frac{a_{2}\left(F_{R}-a_{1}\right)+b_{2}\left(F_{I}-b_{1}\right)-\left(F_{R}-a_{1}\right)^{2}-\left(F_{I}-b_{1}\right)^{2}}{-a_{2}\left(F_{I}-b_{1}\right)+b_{2}\left(F_{R}-a_{1}\right)} .
$$


Furthermore, (13) can be transformed into a formula of $2 \mathrm{D}$ circle:

$$
\begin{gathered}
\left(F_{R}-c_{r}\right)^{2}+\left(F_{I}-c_{j}\right)^{2}=r_{c}^{2}, \\
c_{r}=\frac{-b_{2} R_{0}+2 a_{1} X_{0}+a_{2} X_{0}}{2 X_{0}}, \\
c_{j}=\frac{a_{2} R_{0}+2 b_{1} X_{0}+b_{2} X_{0}}{2 X_{0}}, \\
r_{c}^{2}=\frac{\left(a_{2}^{2}+b_{2}^{2}\right)\left(R_{0}^{2}+X_{0}^{2}\right)}{4 X_{0}^{2}},
\end{gathered}
$$

where $c_{r}$ and $c_{j}$ are the real part and imaginary part of the circle center coordinates, respectively. $r_{c}$ is the radius. It is confirmed that $c_{r}, c_{j}$, and $r_{c}$ are independent of the parametric change of the failure component $P$. Therefore, fault loci fitting requires a small amount of simulation on potential faults, for both catastrophic faults and parametric faults. Alternatively, if $Z_{F}$ is assumed to be a pure reactance $X_{F}$, similar conclusion can be reached.

For a CUT with $n$ components $P_{i}(1 \leq i \leq n)$, fault modeling is to determine the model parameters $c_{r i}, c_{j i}$, and $r_{c i}$ for each $P_{i}$ according to (15), where parameters $c_{r i}$, $c_{j i}$, and $r_{c i}$ are corresponding to $c_{r}, c_{j}$, and $r_{c}$, respectively. In most actual analog cases, (14) is hard to derive from transfer function directly. However, it is well known that three sets of distinct data are sufficient to determine a circle. In this regard, simulation is a simple method to establish the circle model in (14). Therefore, in the modeling process, three sets of distinct output data are obtained by parametric sweep simulations, corresponding to three distinct values of each component $P_{i}$. For the whole CUT, $n$ parametric sweep simulations are needed. Three sets of distinct simulation data for each component are assumed as $F_{c}^{0}, F_{c i}^{1}$, and $F_{c i}^{2}$. Since $F_{c}^{0}$ is assumed to be the fault free output, it fits in with all components. The fault modeling process is illustrated in Figure 1. In addition, the following equation is used to calculate the parameters $c_{r i}, c_{j i}$, and $r_{c i}$ for component $P_{i}(1 \leq$ $i \leq n)$ :

$$
\begin{aligned}
& {\left[\operatorname{Re}\left(F_{c}^{0}\right)-c_{r i}\right]^{2}+\left[\operatorname{Im}\left(F_{c}^{0}\right)-c_{j i}\right]^{2}=r_{c i}{ }^{2},} \\
& {\left[\operatorname{Re}\left(F_{c i}^{1}\right)-c_{r i}\right]^{2}+\left[\operatorname{Im}\left(F_{c i}^{1}\right)-c_{j i}\right]^{2}=r_{c i}{ }^{2},} \\
& {\left[\operatorname{Re}\left(F_{c i}^{2}\right)-c_{r i}\right]^{2}+\left[\operatorname{Im}\left(F_{c i}^{2}\right)-c_{j i}\right]^{2}=r_{c i}{ }^{2} .}
\end{aligned}
$$

2.2. Example of a Sallen-Key Filter. Compared with the difficulty of obtaining explicit mathematical expression, simulation is a relatively easy way for fault modeling. To demonstrate the method, a second-order Sallen-Key filter, as shown in Figure 2, is adopted as an example of circuit under test (CUT). By parameter sweeping simulations with respect to the supply current $I_{d d}$ and the output response $U_{o}$ in PSPICE, the 2D collaborative fault model can be achieved. The simulation results are listed in Table 1, in which parametric ratio means the ratio between failure value and nominal value for each

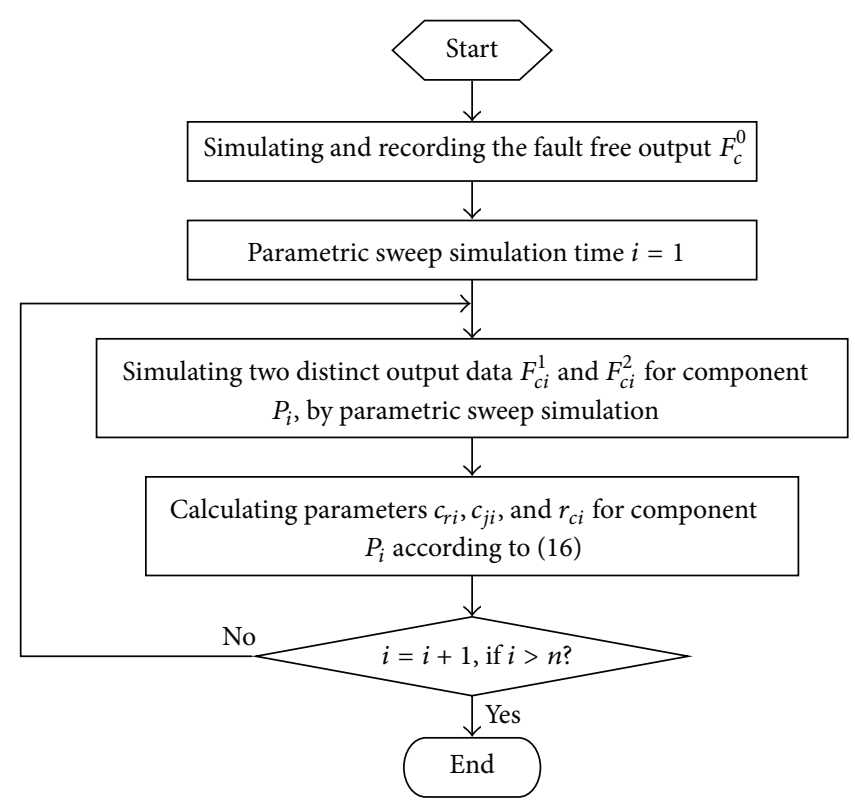

FIGURE 1: Flowchart of fault modeling.

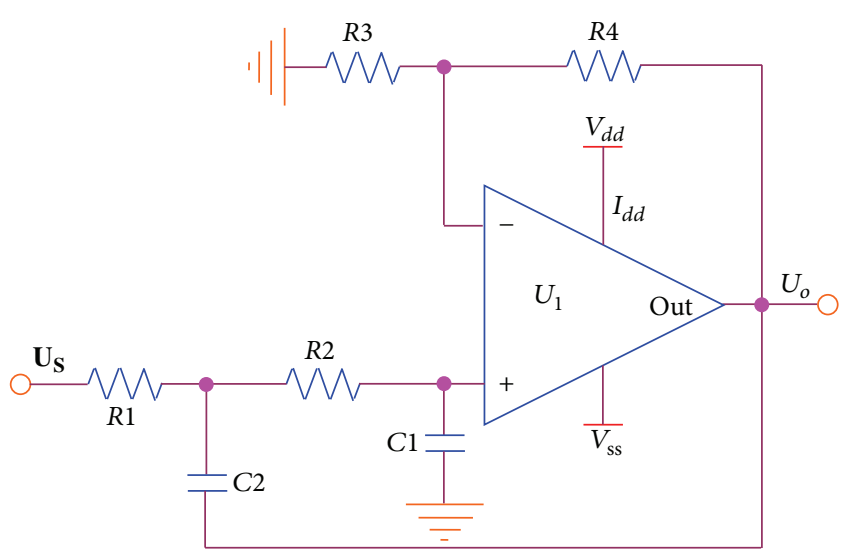

FIgURE 2: Second-order Sallen-key filter, where $R 1, R 2, R 3$ and $R 4$ are $10 \mathrm{k} \Omega, C 1$ and $C 2$ are $4.7 \mathrm{nF}$.

component. Further results are also provided in Figure 3. The stimulation is a $3 \mathrm{kHz}, 1 \mathrm{~V}$ sine signal, and the parameter sweeping range for each component is assumed to be from $p_{i} \times 10^{-4}$ to $p_{i} \times 10^{4}$, where $p_{i}(1 \leq i \leq n)$ is the fault-free value of the $i$ th component $F_{i}$. In addition, the simulation range of $R 3$ and $R 4$ is reduced, because output power of the filter is limited. As shown in Figure 3, all potential fault statuses compose a family of loci on the complex plane, which is called $F_{i}$-loci. Every $F_{i}$-locus corresponds to all the potential fault statuses of a component, not only parametric faults but also catastrophic faults. Furthermore, $F_{i}$-loci converges in the fault free point $A$ and zero point $B$.

Note that in some cases, distances between some $F_{i}$-loci are very small. In extreme cases, some $F_{i}$-loci may coincide with each other, which results in ambiguity group. Since the 2D collaborative fault model is derived from transfer function of CUT, ambiguity group may be an inevitable problem [15]. Table 2 lists the ambiguity groups of the Sallen-Key filter, and 
TABLE 1: Simulation results of 2D model for the Sallen-Key filter.

\begin{tabular}{lcccccc}
\hline $\begin{array}{l}\text { Parametric } \\
\text { ratio }\end{array}$ & $F_{C}(R 1)$ & $F_{C}(R 2)$ & Collaborative simulation results & $F_{C}(C 2)$ \\
\hline 0.01 & $0.7977-j 1.0218$ & $1.7383-j 0.3307$ & - & $-0.0364-j 0.4066$ & $1.1249+j 0.6892$ & $0.2792-j 0.8047$ \\
0.05 & $0.7959-j 1.0537$ & $1.7801-j 0.4091$ & - & $-0.0369-j 0.4382$ & $1.2076+j 0.6979$ & $0.2816-j 0.8265$ \\
0.08 & $0.7925-j 1.0777$ & $1.8094-j 0.4733$ & - & $-0.0373-j 0.4627$ & $1.2755+j 0.7012$ & $0.2830-j 0.8264$ \\
0.1 & $0.7906-j 1.0949$ & $1.8271-j 0.5187$ & - & $-0.0375-j 0.4794$ & $1.3282+j 0.7019$ & $0.2839-j 0.8519$ \\
0.4 & $0.7063-j 1.3735$ & $1.7917-j 1.4096$ & - & $-0.0348-j 0.7801$ & $2.4864+j 0.2002$ & $0.2905-j 1.0578$ \\
0.7 & $0.4829-j 1.6766$ & $0.9513-j 2.0720$ & $0.7186-j 3.9260$ & $-0.0105-j 1.2155$ & $1.9996-j 2.3457$ & $0.2565-j 1.3783$ \\
0.9 & $0.2312-j 1.8427$ & $0.3137-j 2.0195$ & $0.1476-j 2.2588$ & $0.0354-j 1.6315$ & $0.4333-j 2.2252$ & $0.1647-j 1.7011$ \\
1.0 & $0.0767-j 1.8977$ & $0.0767-j 1.8977$ & $0.0767-j 1.8977$ & $0.0767-j 1.8977$ & $0.0767-j 1.8977$ & $0.0767-j .8977$ \\
1.1 & $-0.9320-j 1.9275$ & $-0.1008-j 1.7530$ & $0.0385-j 1.6543$ & $0.1390-j 2.2194$ & $-0.1113-j 1.6025$ & $-0.0746-j 2.1441$ \\
1.3 & $-0.4407-j 1.8928$ & $-0.3146-j 1.4630$ & $0.0013-j 1.3453$ & $0.3832-j 3.0993$ & $-0.2476-j 1.1936$ & $-0.6829-j 2.6877$ \\
1.5 & $-0.7474-j 1.7426$ & $-0.4093-j 1.2169$ & $-0.0151-j 1.1573$ & $1.0301-j 4.5183$ & $-0.2751-j 0.9275$ & $-2.0075-j 2.9003$ \\
2 & $-1.1278-j 1.1435$ & $-0.4420-j 0.8107$ & $-0.0304-j 0.9029$ & $15.264-j 3.1208$ & $-0.2445-j 0.5844$ & $-2.8027+j 0.5055$ \\
3 & $-0.9616-j 0.3874$ & $-0.3514-j 0.4570$ & $-0.0366-j 0.7040$ & - & $-0.1726-j 0.3330$ & $-0.7652+j 0.6990$ \\
5 & $-0.5445-j 0.0583$ & $-0.2246-j 0.2339$ & $-0.0379-j 0.5686$ & - & $-0.1030-j 0.1748$ & $-0.2644+j 0.3346$ \\
10 & $-0.2431+j 0.0129$ & $-0.1143-j 0.1029$ & $-0.0375-j 0.4793$ & - & $-0.0509-j 0.0800$ & $-0.0979+j 0.1423$ \\
30 & $-0.0739+j 0.0104$ & $-0.0383-j 0.0314$ & $-0.0367-j 0.4248$ & - & $-0.0168-j 0.0252$ & $-0.0273+j 0.0425$ \\
100 & $-0.0215+j 0.0036$ & $-0.0115-j 0.0091$ & $-0.0364-j 0.4067$ & - & $-0.0050-j 0.0074$ & $-0.0078+j 0.0124$ \\
\hline & & & & & - &
\end{tabular}

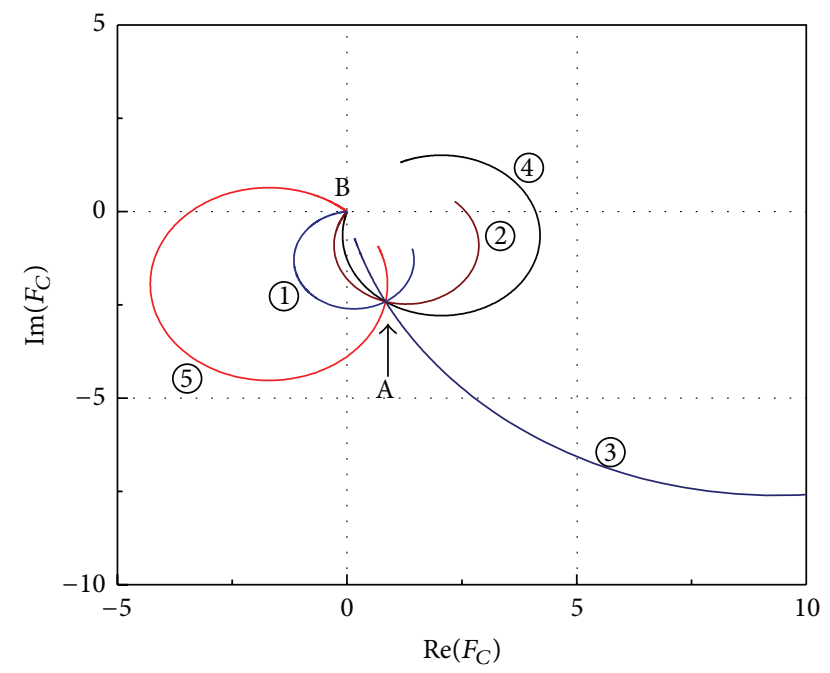

FIGURE 3: 2D collaborative fault model of the Sallen-Key filter. All potential fault statuses of the filter compose a family of $F_{i}$-loci, and $F_{i}$-loci converge in the fault free point $\mathrm{A}$ and zero point $\mathrm{B}$.

Figure 3 shows that while the $F_{i}$-loci of $R 1, R 2, C 1$, and $C 2$ are obviously different from others, fault status of $R 3$ and $R 4$ are located on the same locus. This means that potential faults of $R 3$ and $R 4$ cannot be isolated based on the fault model in Figure 3; therefore, $R 3$ and $R 4$ fall into the same ambiguity group $\{R 3, R 4\}$.

\section{Fault Modeling in 3D Complex Space}

Based on the measurements of supply current and voltage response at any test point, the $2 \mathrm{D}$ collaborative fault model
TABLE 2: Ambiguity groups of the Sallen-Key filter.

\begin{tabular}{lc}
\hline Ambiguity group & Fault components \\
\hline (1) & $R 1$ \\
$(2)$ & $R 2$ \\
(3) & $R 3, R 4$ \\
(4) & $C 1$ \\
(5) & $C 2$ \\
\hline
\end{tabular}

transforms all potential failure statuses into a family of loci. Therefore, both parametric faults and catastrophic faults can all be detected and located. However, due to the limits of component tolerance and measurement error, the 2D fault model is difficult to apply in actual analog circuits. As depicted in Figure 3, between point $\mathrm{A}$ and point $\mathrm{B}$, the $R 2$ and $\mathrm{C1}$-loci are too close to be distinguished. Therefore, to increase the practicability for actual applications, the $2 \mathrm{D}$ fault model is extended into 3D complex space.

3.1. Theorem of 3D Collaborative Fault Model. The 2D function in (3) is a linear combination of supply current $I_{d d}$ and voltage response $U_{o}$, based on the measurements of real part $\operatorname{Re}(\cdot)$ and imaginary part $\operatorname{Im}(\cdot)$ of each complex variable. $I_{d d}$ and $U_{o}$ are easier to measure in-circuit from limited test points than other circuit variables, such as the input admittance. Furthermore, the complex modulus (absolute value) $|\cdot|$ is also employed to compose the $3 \mathrm{D}$ function. This helps to increase the distance between $F_{i}$-loci fault models and improve the FIR.

Similarly, the component $P$ in (2) is still assumed to be the failure component. Therefore, by defining $x, y$, and $z$ as 
TABLE 3: Simulation results for $R 2$ and $C 1$ in $3 D$ model.

\begin{tabular}{lcccccc}
\hline \multirow{2}{*}{ Parametric ratio } & \multicolumn{3}{c}{ Simulation results of $R 2$} & \multicolumn{3}{c}{ Simulation results of $C 1$} \\
& $F_{U}(R 2)$ & $F_{I D D}(R 2)$ & $c_{1} I_{d d}$ & $F_{U}(C 1)$ & $F_{I D D}(C 1)$ & $c_{1} I_{d d}$ \\
\hline 0.01 & $2.052-j 0.040$ & 2.3371 & $-0.3137-j 0.2910$ & $1.156+j 1.009$ & 3.1270 & $-0.0276-j 0.3186$ \\
0.05 & $2.115-j 0.121$ & 2.2642 & $-0.3349-j 0.2879$ & $1.251+j 1.034$ & 2.9564 & $-0.0380-j 0.3361$ \\
0.08 & $2.161-j 0.189$ & 2.116 & $-0.3516-j 0.2843$ & $1.328+j 1.051$ & 2.8331 & $-0.0472-j 0.3498$ \\
0.1 & $2.190-j 0.238$ & 2.1782 & $-0.3629-j 0.2812$ & $1.382+j 1.061$ & 2.7540 & $-0.0538-j 0.3591$ \\
0.4 & $2.323-j 1.263$ & 1.8144 & $-0.5313-j 0.1466$ & $2.804+j 0.713$ & 1.6581 & $-0.3176-j 0.5127$ \\
0.7 & $1.491-j 2.184$ & 1.8142 & $-0.5397+j 0.1120$ & $2.743-j 2.293$ & 1.3418 & $-0.7434-j 0.0527$ \\
0.9 & $0.752-j 2.248$ & 2.0242 & $-0.4380+j 0.2285$ & $0.929-j 2.460$ & 1.8244 & $-0.4953+j 0.2348$ \\
1.0 & $0.457-j 2.155$ & 2.1775 & $-0.3804+j 0.2573$ & $0.457-j 2.155$ & 2.1775 & $-0.3804+j 0.2573$ \\
1.1 & $0.226-j 2.024$ & 2.3555 & $-0.3268+0.2710$ & $0.185-j 1.854$ & 2.5751 & $-0.2959+j 0.2515$ \\
1.3 & $-0.075-j 1.734$ & 2.7645 & $-0.2396+j 0.2710$ & $-0.051-j 1.413$ & 3.3929 & $-0.1970+j 0.2194$ \\
1.5 & $-0.231-j 1.471$ & 3.2227 & $-0.1781+j 0.2541$ & $-0.134-j 1.114$ & 4.2771 & $-0.1410+j 0.1865$ \\
2 & $-0.348-j 1.013$ & 4.4787 & $-0.0945+j 0.2023$ & $-0.166-j 0.716$ & 6.5293 & $-0.0787+j 0.1314$ \\
3 & $-0.313-j 0.591$ & 7.1763 & $-0.0386+j 0.1339$ & $-0.133-j 0.414$ & 11.0258 & $-0.0401+j 0.0814$ \\
5 & $-0.211-j 0.311$ & 12.7565 & $-0.0133+j 0.0773$ & $-0.084-j 0.220$ & 20.3875 & $-0.0193+j 0.0451$ \\
10 & $-0.111-j 0.140$ & 26.8834 & $-0.0036+j 0.0370$ & $-0.043-j 0.101$ & 43.6119 & $-0.0083+j 0.0214$ \\
30 & $-0.038-j 0.043$ & 83.5634 & $-0.0006+j 0.0120$ & $-0.014-j 0.032$ & 136.5537 & $-0.0025+j 0.0069$ \\
100 & $-0.011-j 0.013$ & 281.8780 & $-0.0001+j 0.0035$ & $-0.004-j 0.009$ & 461.8366 & $-0.0007+j 0.0020$ \\
\hline & & & & & &
\end{tabular}

the unit vectors in $3 \mathrm{D}$ complex space, a transformation function $F_{3 \mathrm{D}}^{1}(\cdot)$ can be composed as follow [14]:

$$
\begin{aligned}
F_{3 \mathrm{D}}^{1}(P)= & x \cdot \operatorname{Re}\left[F_{U}(P)\right] \\
& +y \cdot \operatorname{Im}\left[F_{U}(P)\right]+z \cdot\left|\frac{1}{F_{I D D}(P)}\right|,
\end{aligned}
$$

where

$$
\begin{gathered}
F_{U}(P)=c_{0} U_{o}, \\
F_{I D D}(P)=\frac{1}{\left(c_{1} I_{d d}\right)},
\end{gathered}
$$

where $c_{0}$ and $c_{1}$ are still adjustment coefficients as in (3). Taking the component pair $\mathrm{R} 2-\mathrm{C} 1$ as an example, the distance $D_{3 \mathrm{D}}^{1}(\cdot)$ between $R 2$ and $C 1$-loci is

$$
\begin{aligned}
& D_{3 \mathrm{D}}^{1}(R 2, C 1) \\
& \quad=\sqrt{\left[D_{x}^{1}(R 2, C 1)\right]^{2}+\left[D_{y}^{1}(R 2, C 1)\right]^{2}+\left[D_{z}^{1}(R 2, C 1)\right]^{2}},
\end{aligned}
$$

where $D_{x}^{1}(\cdot), D_{y}^{1}(\cdot)$, and $D_{z}^{1}(\cdot)$ are distances corresponding to each axe in $3 \mathrm{D}$ complex space:

$$
\begin{aligned}
& D_{x}^{1}(R 2, C 1)=\left|\operatorname{Re}\left[F_{U}(R 2)\right]-\operatorname{Re}\left[F_{U}(C 1)\right]\right|, \\
& D_{y}^{1}(R 2, C 1)=\left|\operatorname{Im}\left[F_{U}(R 2)\right]-\operatorname{Im}\left[F_{U}(C 1)\right]\right|, \\
& D_{z}^{1}(R 2, C 1)=|| \frac{1}{F_{I D D}(R 2)}|-| \frac{1}{F_{I D D}(C 1)} \| .
\end{aligned}
$$

Although the distance $D_{3 \mathrm{D}}^{1}(\cdot)$ in $(20)$ has been proved to be better than that in 2D F $F_{i}$-loci fault model [14], it is calculated based on voltage and input admittance, whose absolute values are usually far greater than supply current. Therefore, for an appropriate coefficient $c_{1}$, the following inequality can be guaranteed:

$$
\left|\frac{1}{F_{I D D}(P)}\right|=\left|c_{1} I_{d d}\right|<1 \text {. }
$$

In addition, a new distance $D_{z}^{2}(\cdot)$ is defined as follows:

$$
\begin{aligned}
D_{z}^{2}(R 2, C 1) & =\left\|F_{I D D}(R 2)|-| F_{I D D}(C 1)\right\|, \\
D_{z}^{2}(R 2, C 1) & =\frac{D_{z}^{1}(R 2, C 1)}{1 /\left|F_{I D D}(R 2)\right| \cdot 1 /\left|F_{I D D}(C 1)\right|} \\
& >D_{z}^{1}(R 2, C 1) .
\end{aligned}
$$

Therefore, a new transformation function $F_{3 \mathrm{D}}^{2}(\cdot)$ for the 3D fault model can be defined as

$$
F_{3 \mathrm{D}}^{2}(P)=x \cdot \operatorname{Re}\left[F_{U}(P)\right]+y \cdot \operatorname{Im}\left[F_{U}(P)\right]+z \cdot\left|F_{I D D}(P)\right| .
$$

Furthermore, the distance $D_{3 \mathrm{D}}^{2}(\cdot)$ is defined for the new function $F_{3 \mathrm{D}}^{2}(\cdot)$. Taking $R 2$ and $C 1$ as an example, it can be expressed as

$$
\begin{aligned}
& D_{3 \mathrm{D}}^{2}(R 2, C 1) \\
& \quad=\sqrt{\left[D_{x}^{1}(R 2, C 1)\right]^{2}+\left[D_{y}^{1}(R 2, C 1)\right]^{2}+\left[D_{z}^{2}(R 2, C 1)\right]^{2}} .
\end{aligned}
$$

3.2. Simulation Example of Sallen-Key Filter for the 3D Model. For a given input of $1 \mathrm{~V}, 3 \mathrm{kHz}$ sine signal, PSPICE results on the Sallen-Key filter are obtained. The key part of simulation results are listed in Table 3 . The original simulation results 


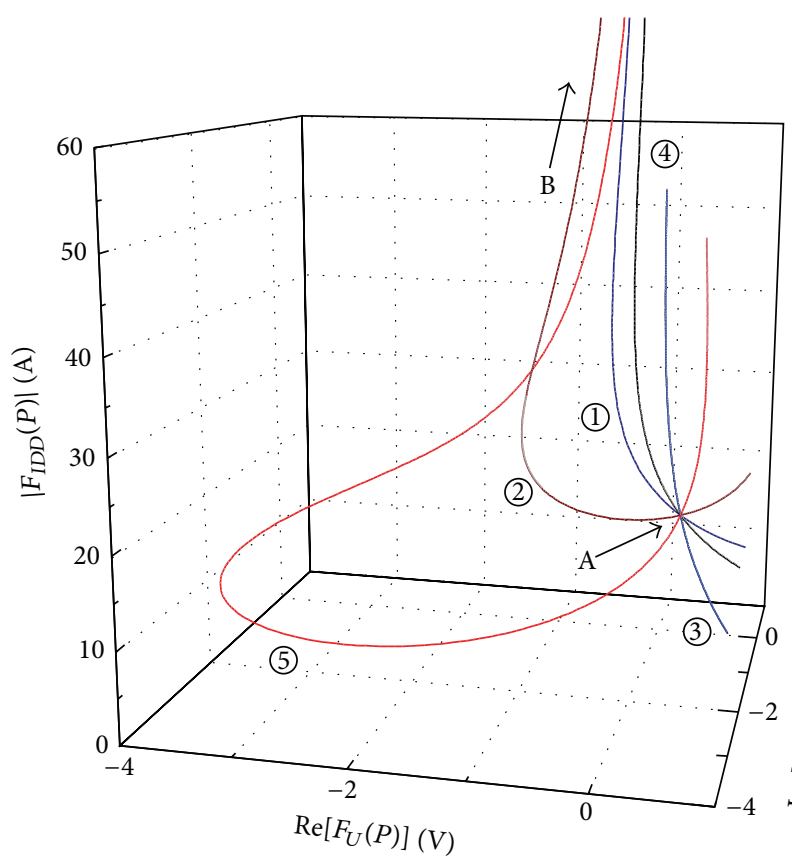

FIGURE 4: Family of fault loci in 3D complex place for the Sallen-Key filter. All $F_{i}$-loci converge in the fault free point A, while some $F_{i}$-loci have tendency to an infinity point $\mathrm{B}$.

include $U_{o}$ and $I_{d d}$, corresponding to all potential failure status of each component, such as $R 2$ and $C 1$, and then all the data in Table 3 is obtained according to (18) and (19). Moreover, the simulation results are also illustrated integrally in Figure 4. It shows that all $F_{i}$-loci converge in the fault free point $\mathrm{A}$, which is similar to the $2 \mathrm{D} F_{i}$-loci. However, due to the definition in (19), some $F_{i}$-loci tend to be an infinity point $\mathrm{B}$, instead of the determinate point $\mathrm{B}$ in $2 \mathrm{D}$ model. The associated ambiguity groups are the same as shown in Table 2.

To validate the improvement of fault model $F_{3 \mathrm{D}}^{2}(\cdot)$ in $(24)$, the $2 \mathrm{D}$ model proposed in Section 2 is used as a reference. In addition, both the 3D model $F_{3 \mathrm{D}}^{1}(\cdot)$ proposed in [14], and the complex-circle-based fault model proposed in [12, 13] are adopted for comparison. In the example of $R 2$ and $C 1$ in the Sallen-Key filter, distances between $R 2$ and $C 1$ loci are provided in Table 4, according to each fault model, respectively; the comparison range is from $p_{i} \times 10^{-2}$ to $p_{i} \times 10^{2}$, where $p_{i}$ is the fault-free value of $R 2$ and $C 1$. The results of comparison between different methods are also illustrated in Figure 5 , in which the curve $d 1$ is the distance of $R 2-C 1$ in the $3 \mathrm{D}$ model $F_{3 \mathrm{D}}^{2}(\cdot)$, the curve $d 2$ is the distance defined in the $2 \mathrm{D}$ model, the dash curve $d 3$ is defined by $F_{3 \mathrm{D}}^{1}(\cdot)[14]$, and dash curve $d 4$ is related to complex-circle-based fault model $[12,13]$. As shown in Table $4, d 3$ is better than $d 4$; however, because the biggest gap between $d 3$ and $d 4$ is less than $2.2 \%$, $d 4$ is very close to $d 3$ in Figure 5 . Moreover, $d 1$ is much better than all the others. Since $d 3$ is also based on the 3D model in [14], it is taken as an ideal reference. On average, $d 1$ is $9.6 \%$ bigger than $d 3$, in the value range from $p_{i} \times 10^{-2}$ to $p_{i}$. Moreover, in the range from $p_{i}$ to $p_{i} \times 10^{2}$, because $I_{d d}$ and $U_{o}$ tend to be zero, $d 1$ increases continuously while $d 3$ reduces,
TABLE 4: Distances between $R 2$ and C1-loci with respect to different fault models.

\begin{tabular}{lcccc}
\hline Parametric ratio & $d 1$ & $d 2$ & $d 3$ & $d 4$ \\
\hline 0.01 & 1.5896 & 1.1902 & 1.3836 & 1.3805 \\
0.05 & 1.6000 & 1.2463 & 1.4463 & 1.4453 \\
0.08 & 1.6180 & 1.2902 & 1.4971 & 1.4963 \\
0.1 & 1.6342 & 1.3184 & 1.5324 & 1.5294 \\
0.4 & 2.0396 & 1.7533 & 2.0343 & 2.0336 \\
0.7 & 1.3426 & 1.0835 & 1.2716 & 1.2567 \\
0.9 & 0.3408 & 0.2379 & 0.2814 & 0.2761 \\
1.0 & 0 & 0 & 0 & 0 \\
1.1 & 0.2808 & 0.1509 & 0.1787 & 0.1750 \\
1.3 & 0.7061 & 0.2776 & 0.3288 & 0.3219 \\
1.5 & 1.1175 & 0.3190 & 0.3778 & 0.3700 \\
2 & 2.0800 & 0.3004 & 0.3553 & 0.3483 \\
3 & 3.8577 & 0.2176 & 0.2570 & 0.2523 \\
5 & 7.6327 & 0.1353 & 0.1596 & 0.1568 \\
10 & 16.7287 & 0.0675 & 0.0795 & 0.0782 \\
30 & 52.9903 & 0.0223 & 0.0263 & 0.0259 \\
100 & 179.9586 & 0.0067 & 0.0079 & 0.0077 \\
\hline
\end{tabular}

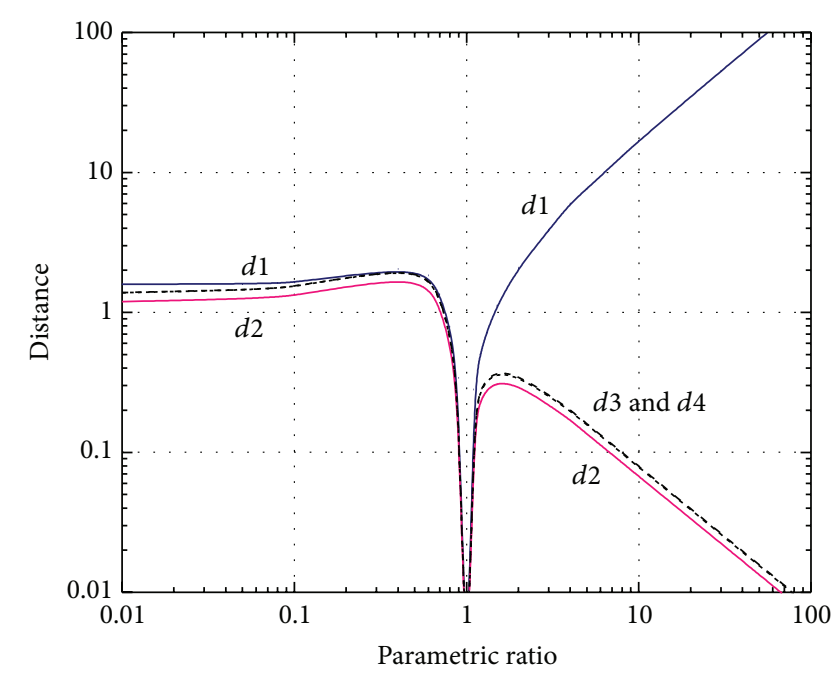

FIGURE 5: Distances between $R 2$ and $C 1$-loci with respect to different fault models. Curve $d 1$ is defined by the $3 \mathrm{D}$ model proposed in Section $3, d 2$ is the $2 \mathrm{D}$ model proposed in Section 2, $d 3$ is corresponding to the 3D model proposed in [14], and $d 4$ is corresponding to the complex-circle-based fault model proposed in $[12,13]$.

and the advantage of $d 1$ is more significant. Thus, 3D fault model $F_{3 \mathrm{D}}^{2}(\cdot)$ results in better FIR against the measurement errors and parametric tolerance.

\section{DFT by Adding Switched Bypass-Components}

4.1. Theory of Improved DFT Method. A common theory of ambiguity group in fault diagnosis has been proposed in previous research [15]. If the sensitivities of some ana$\log$ components are linearly dependent on all frequencies, 
the change of these components cannot be distinguished, and thus these components fall into an ambiguity group. Based on this theory, components $R_{a}$ and $R_{b}$ in an analog CUT are assumed to fall into the ambiguity groups $\left\{R_{a}, R_{b}\right\}$; similar to $R 3$ and $R 4$ of the Sallen-Key filter in Figure 2, a linear ratio $r$ is defined as

$$
r=\frac{R_{b}}{R_{a}} .
$$

In addition, $H(s)$ is assumed to be the transfer function of CUT and it is a function of $r$ as

$$
H(s)=f(r),
$$

where $s$ is the Laplacian operator. Therefore,

$$
\begin{gathered}
\frac{\partial H(s)}{\partial R_{a}}=\frac{\partial f}{\partial r} \frac{\partial r}{\partial R_{a}}=\frac{\partial f}{\partial r}\left(-\frac{R_{b}}{R_{a}^{2}}\right), \\
\frac{\partial H(s)}{\partial R_{b}}=\frac{\partial f}{\partial r} \frac{\partial r}{\partial R_{b}}=\frac{\partial f}{\partial r} \frac{1}{R_{a}}=-\frac{1}{r} \frac{\partial H(s)}{\partial R_{a}} .
\end{gathered}
$$

Equation (29) shows the sensitivity dependence between $R_{a}$ and $R_{b}$, which results in the ambiguity group $\left\{R_{a}, R_{b}\right\}$. Therefore, transforming the topological structure of CUT and breaking the linear sensitivity dependence $r$ should be an effective DFT method. One of the most common DFT methods is bypassing and the traditional bypassing method is based on reducing the capacitive effects [16-18]. However, for the 3D model in complex space, keeping or increasing the capacitive effects is expected to be a far more effective method.

For the purpose of increasing the capacitive effects, $C_{b}$ is assumed to be added in CUT as a parallel branch of $R_{b}$, and then the impedance $Z_{b}$ of the parallel branch is

$$
Z_{b}=\frac{R_{b} \times 1 / s C_{b}}{R_{b}+1 / s C_{b}}=\frac{R_{b}}{1+s C_{b} R_{b}} .
$$

Thus the new linear ratio $r^{\prime}$ is defined as

$$
r^{\prime}=\frac{Z_{b}}{R_{a}}=\frac{R_{b}}{R_{a}\left(1+s C_{b} R_{b}\right)} .
$$

So the corresponding transfer function $H^{\prime}(s)$ is defined based on function $f(\cdot)$ :

$$
\begin{gathered}
H^{\prime}(s)=f\left(r^{\prime}\right), \\
\frac{\partial H^{\prime}(s)}{\partial R_{a}}=\frac{\partial f}{\partial r^{\prime}} \frac{\partial r^{\prime}}{\partial R_{a}}=\frac{\partial f}{\partial r^{\prime}} \frac{R_{b}}{\left(1+s C_{b} R_{b}\right)} \frac{-1}{R_{a}^{2}}, \\
\frac{\partial H^{\prime}(s)}{\partial R_{b}}=\frac{\partial f}{\partial r^{\prime}} \frac{\partial r^{\prime}}{\partial R_{b}}=\frac{\partial f}{\partial r^{\prime}} \frac{1}{R_{a}} \frac{1}{\left(1+s C_{b} R_{b}\right)^{2}}, \\
\frac{\partial H^{\prime}(s)}{\partial C_{b}}=\frac{\partial f}{\partial r^{\prime}} \frac{\partial r^{\prime}}{\partial C_{b}}=\frac{\partial f}{\partial r^{\prime}} \frac{R_{b}}{R_{a}} \frac{-s R_{b}}{\left(1+s C_{b} R_{b}\right)^{2}} ;
\end{gathered}
$$

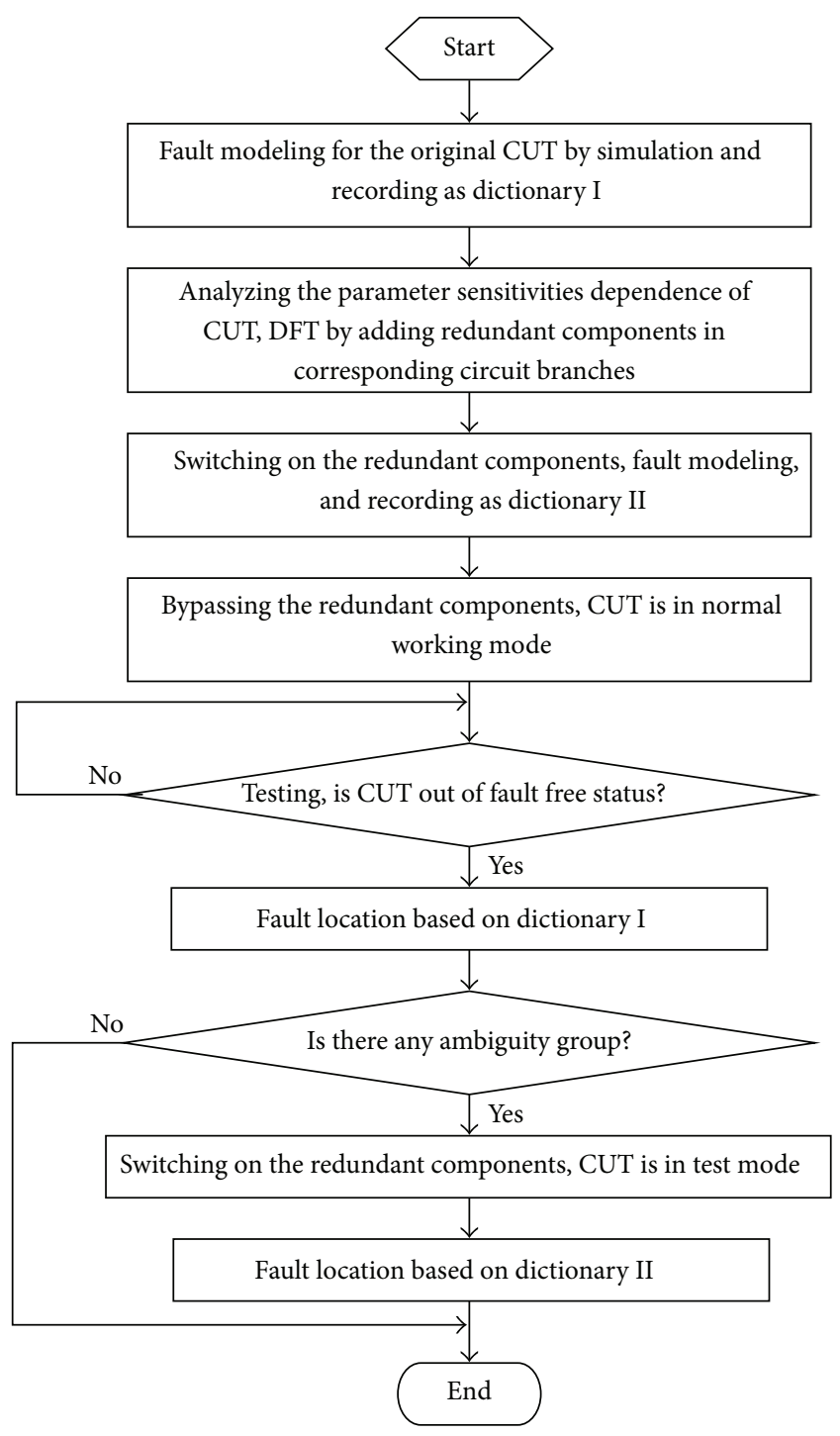

FIGURE 6: Flowchart of bypassing-based DFT and testing.

then

$$
\begin{gathered}
\frac{\partial H^{\prime}(s) / \partial R_{a}}{\partial H^{\prime}(s) / \partial R_{b}}=\frac{-R_{b}\left(1+s C_{b} R_{b}\right)}{R_{a}}, \\
\frac{\partial H^{\prime}(s) / \partial R_{a}}{\partial H^{\prime}(s) / \partial C_{b}}=\frac{s R_{b}\left(1+s C_{b} R_{b}\right)}{R_{a}}, \\
\frac{\partial H^{\prime}(s) / \partial R_{b}}{\partial H^{\prime}(s) / \partial C_{b}}=\frac{-1}{s R_{b}{ }^{2}} .
\end{gathered}
$$

As shown in (33), the parameter sensitivities for $R_{a}, R_{b}$, and $C_{b}$ are not linear obviously, which means that their faults can be isolated. In a similar way, if $R_{a}$ and $C_{b}$ fall into the same ambiguity group because their sensitivities are linearly dependent, some $R_{b}$ can be paralleled with $C_{b}$ for DFT purpose. Therefore, if the status of CUT is partitioned into working mode and testing mode, the DFT and testing flow proposed in previous can be illustrated in Figure 6. 


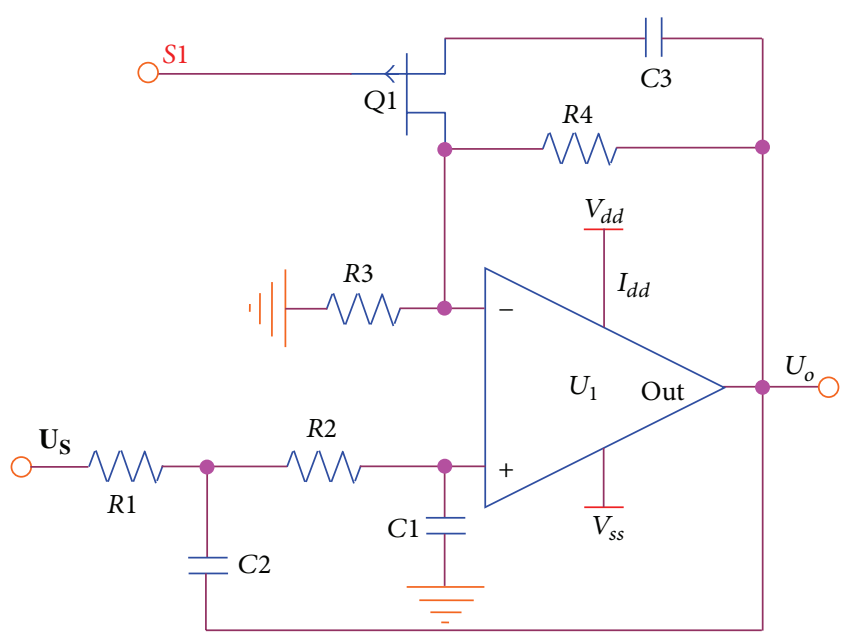

FIgURE 7: The Sallen-Key filter after adding switched bypasscomponent C3.C3 is added for the purpose of DFT, and MOS switch Q1 is used to bypass C3 in filter mode.

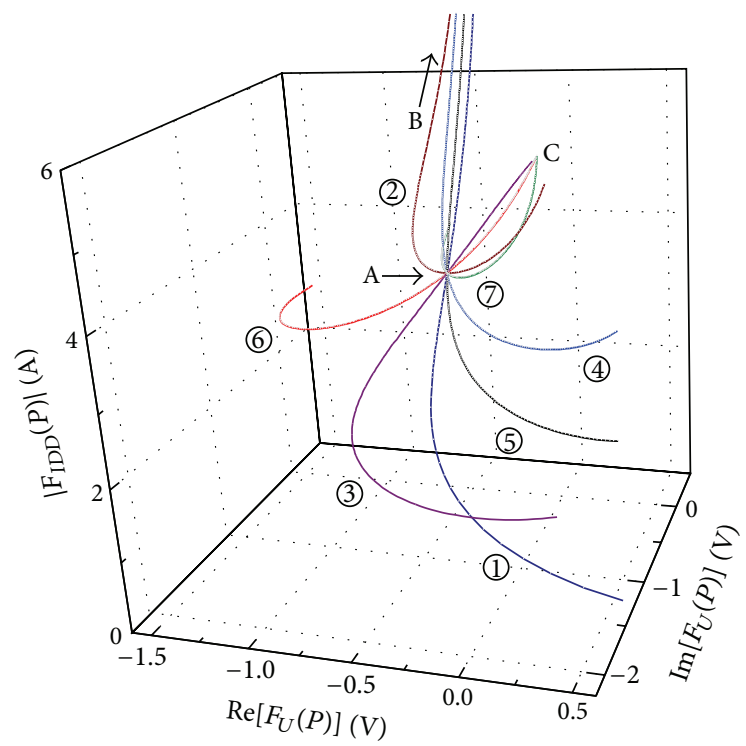

Figure 8: 3D fault model of the Sallen-key filter by DFT. Point A means the fault free status of all $F_{i}$-loci, and some $F_{i}$-loci are tendency to an infinity point $\mathrm{B}$.

4.2. Example and Simulation on Sallen-Key Filter. In the Sallen-Key filter, not only the collaborative fault model on 2D Complex Plane but also the $F_{i}$-loci in $3 \mathrm{D}$ complex space show that $R 3$ and $R 4$ fall into an ambiguity group, which is also illustrated in Table 2. Therefore, for the purpose of DFT, the switched bypass-component $C 3$ is added by using a $P$ channel MOS switch Q1 in Figure 7. When the control signal $S 1=1, Q 1$ is turned off, the CUT is in common working mode, and C3 is bypassed by Q1. Otherwise, when $S 1=0$, the CUT is switched into testing mode and C3 is parallel to $R 4$ because $Q 1$ is turned on.

With the input of $1 \mathrm{~V}, 3 \mathrm{kHz}$ sine signal, PSPICE simulation results in Figure 8 shows the $F_{i}$-loci of the Sallen-Key
TABLE 5: Updated ambiguity groups of the Sallen-Key filter by DFT.

\begin{tabular}{lc}
\hline Ambiguity group & Fault components \\
\hline (1) & $R 1$ \\
(2) & $R 2$ \\
(3) & $R 3$ \\
(4) & $R 4$ \\
(5) & $C 1$ \\
(6) & $C 2$ \\
(7) & $C 3$ \\
\hline
\end{tabular}

filter after DFT, where $R 3$ and R4-loci separate obviously. All $F_{i}$-loci converge in the fault free point $\mathrm{A}$, while points $\mathrm{B}$ and $C$ are the other two intersections, and point $B$ is located at infinity theoretically. Furthermore, the updated ambiguity groups are listed in Table 5 and $R 3$ and $R 4$ belong to different ambiguity groups. This means that adding switched bypasscomponents is an effective method to improve the FIR.

In addition, compared with the DFT methods proposed in [16-18], the improved method given in this paper is also more effective to some extent. Both Sun [16] and Hong [17] reduce capacitive effects by bypassing the capacitors in original CUT, to achieve DFT in testing mode; however, this method cannot isolate the ambiguity group like $\{R 3, R 4\}$ in the Sallen-Key filter. The DFT method given in this paper is based on the bypassing approach proposed in [16]. It improves DFT method by adding some redundant components in the CUT and bypassing these components only in working mode. It offers appealing potential to isolate some ambiguity groups compared with the methods in $[16,17]$. Moreover, isolation of both catastrophic faults and parametric faults of analog circuits is possible with the improved DFT method.

\section{Conclusion}

In order to cope with the difficulties of fault diagnosis in analog circuits, a 2D fault model based on collaborative supply current and output voltage is first proposed in this paper. This $2 \mathrm{D}$ fault model is proved to be a family of circles on complex plane and helps to simplify the simulation of potential faults greatly. Then, the fault model is improved in the $3 \mathrm{D}$ complex space, and a family of $F_{i}$-loci is obtained to illustrate the potential faults of each analog component. Since the distances between each $F_{i}$-locus are increased, the 3D model achieves a far better FIR against the measurement error and parametric tolerance. The proposed fault models are validated through examples on a Sallen-Key filter.

However, it is worth noting that ambiguity group is still an inevitable problem. Therefore, to increase the FIR sequentially, an improved bypassing-based DFT method is introduced by using MOS switches and adding some redundant components in the CUT. The simulation results show that, except for few limited failure statuses, the parametric and catastrophic faults in the analog CUT can be isolated and the problem of ambiguity groups is well resolved with improved FIR. The fault models on 2D complex plane and 
3D space are all based on the assumption of single fault; thus the improved bypassing methods proposed in this paper are also limited to single fault. In the presence of multifaults, this fault model becomes complicated and thus further research effort is needed in this area.

\section{Conflict of Interests}

The authors declare that there is no conflict of interests regarding the publication of this paper.

\section{Acknowledgments}

This research was supported in part by NSFC (60934002 and 61071029) and Key Laboratory Project (YQ15103 and YQ15116).

\section{References}

[1] A. S. Sarathi Vasan, B. Long, and M. Pecht, "Diagnostics and prognostics method for analog electronic circuits," IEEE Transactions on Industrial Electronics, vol. 60, no. 11, pp. 52775291, 2013.

[2] P. Wang and S. Y. Yang, "A soft fault dictionary method for analog circuit diagnosis based on slope fault mode," Control and Automation, vol. 22, no. 6, pp. 1-23, 2006.

[3] C. L. Yang, S. L. Tian, Z. Liu, J. Huang, and F. Chen, "Fault modeling on complex plane and tolerance handling methods for analog circuits," IEEE Transactions on Instrumentation and Measurement, vol. 62, no. 10, pp. 2730-2738, 2013.

[4] X. Gao, H. J. Wang, and Z. Liu, "Handing tolerance problem in fault diagnosis of linear-analogue circuits with accurate statistics approach," Journal of Applied Mathematics, vol. 2013, Article ID 414120, 9 pages, 2013.

[5] Y. Wang, L. Wang, X. Wang, and J. Ma, "An improved analog circuit fault dictionary," Journal of Beijing University of Chemical Technology, vol. 38, no. 2, pp. 129-133, 2011.

[6] B. Long, S. Tian, and H. Wang, "Feature vector selection method using Mahalanobis distance for diagnostics of analog circuits based on LS-SVM," Journal of Electronic Testing, vol. 28, no. 5, pp. 745-755, 2012.

[7] A. Saha, F. Rahman, R. Al-Maruf, H. Rahman, and A. B. M. H. Rashid, "Detection and localization of faults in analog integrated circuits by utilizing the combined effect of output voltage gain and phase variation with frequency," in Proceedings of the IEEE Region 10 Conference (TENCON '09), pp. 1-5, IEEE, November 2009.

[8] F. Wu, S. Yin, and H. R. Karimi, "Fault detection and diagnosis in process data using support vector machines," Journal of Applied Mathematics, vol. 2014, Article ID 732104, 9 pages, 2014.

[9] I. M. Bell, S. J. spinks, and J. M. da Silva, "Supply current test of analogue and mixed signal circuits," IEE Proceedings-Circuits, Devices and Systems, vol. 143, no. 6, pp. 399-407, 1996.

[10] S. Umezu, M. Hashizume, and H. Yotsuyanagi, "A built-in supply current test circuit for pin opens in assembled PCBs," in Proceedings of the International Conference on Electronics Packaging (ICEP '14), pp. 227-230, Toyama, Japan, April 2014.

[11] L. Ma and H. Wang, "Fault diagnosis method based on output voltage and supply current collaborative analysis," Chinese Journal of Scientific Instrument, vol. 34, no. 8, pp. 1872-1878, 2013.
[12] C. Yang, J. Yang, Z. Liu, and S. Tian, "Complex field fault modeling-based optimal frequency selection in linear analog circuit fault diagnosis," IEEE Transactions on Instrumentation and Measurement, vol. 63, no. 4, pp. 813-825, 2014.

[13] S. L. Tian, C. L. Yang, F. Chen, and Z. Liu, "Circle equationbased fault modeling method for linear analog circuits," IEEE Transactions on Instrumentation and Measurement, vol. 63, no. 9, pp. 2145-2159, 2014.

[14] Z. Czaja and R. Zielonko, "Fault diagnosis in electronic circuits based on bilinear transformation in 3-D and 4-D spaces," IEEE Transactions on Instrumentation and Measurement, vol. 52, no. 1, pp. 97-102, 2003.

[15] G. N. Stenbakken, T. M. Souders, and G. W. Stewart, "Ambiguity groups and testability," IEEE Transactions on Instrumentation and Measurement, vol. 38, no. 5, pp. 941-947, 1989.

[16] Y. Sun and M. Hasan, "Design-for-testability of analogue filters," in Test and Diagnosis of Analogue, Mixed-Signal and RF Integrated Circuits, Y. C. Sun, Ed., chapter 6, pp. 179-189, The Institution of Engineering and Technology, 2008.

[17] H.-C. Hong, "A static linear behavior analog fault model for switched-capacitor circuits," IEEE Transactions on ComputerAided Design of Integrated Circuits and Systems, vol. 31, no. 4, pp. 597-609, 2012.

[18] E. Esfandiari and N. Bin Mariun, "Bypassing the short-circuit faults in the switch-ladder multi-level inverter," in Proceedings of the IEEE Symposium on Industrial Electronics and Applications (ISIEA '11), pp. 128-132, September 2011. 


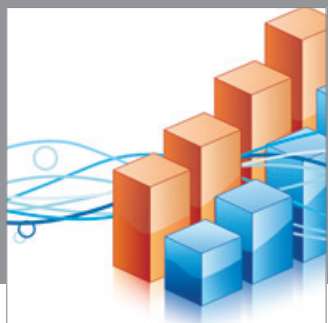

Advances in

Operations Research

mansans

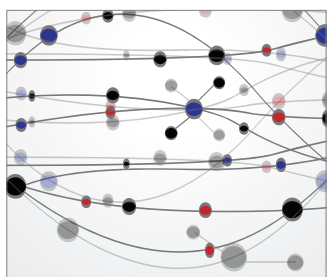

The Scientific World Journal
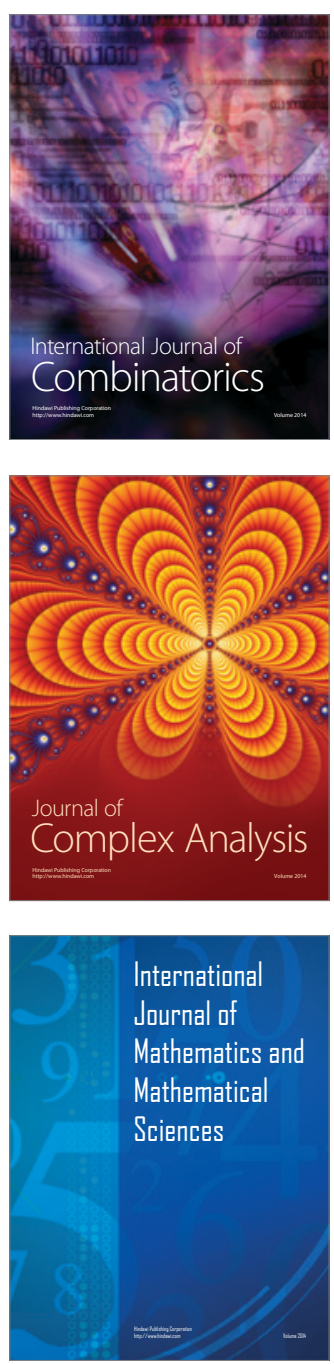
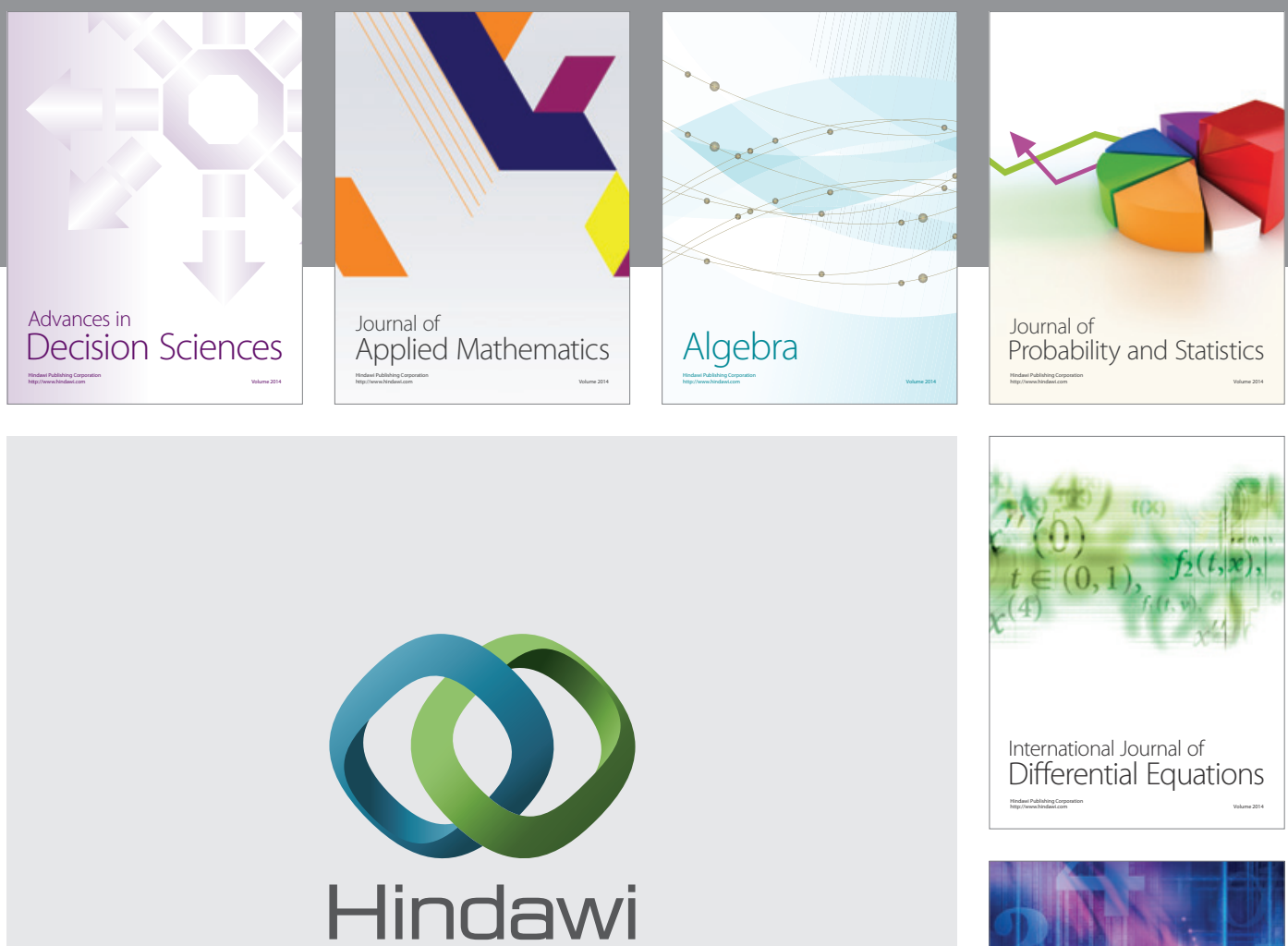

Submit your manuscripts at http://www.hindawi.com
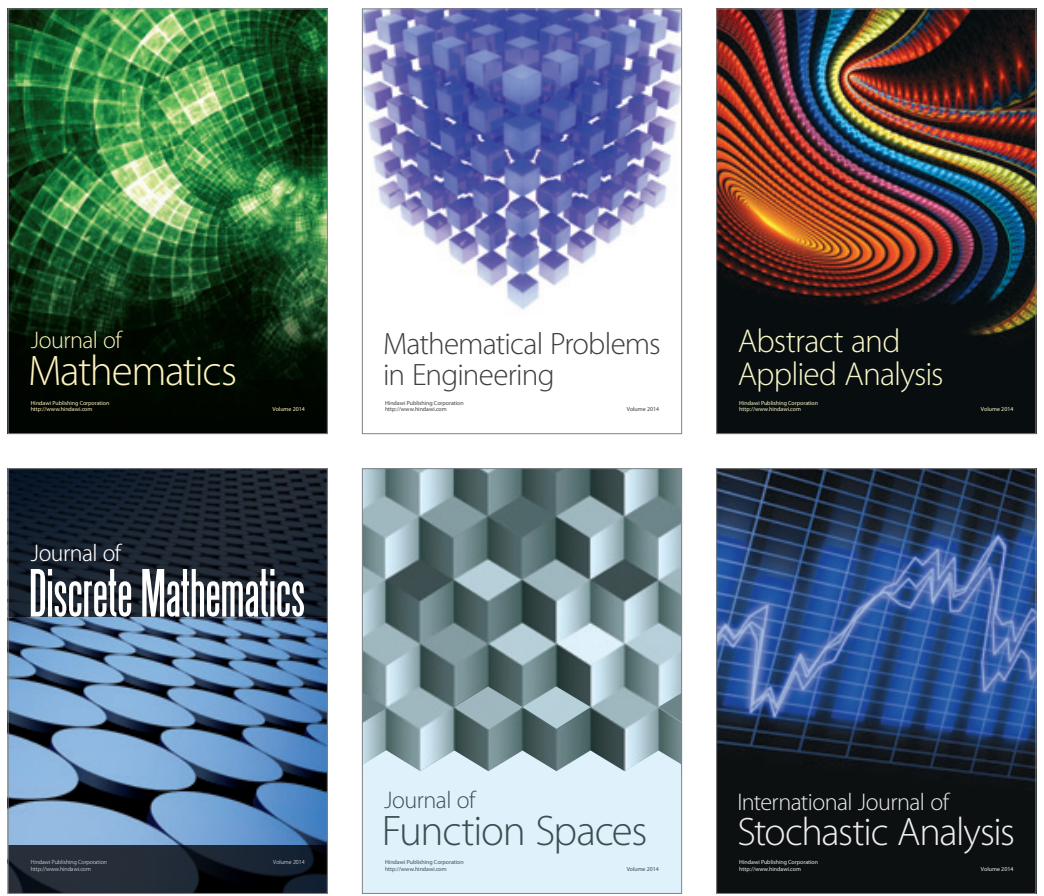

Journal of

Function Spaces

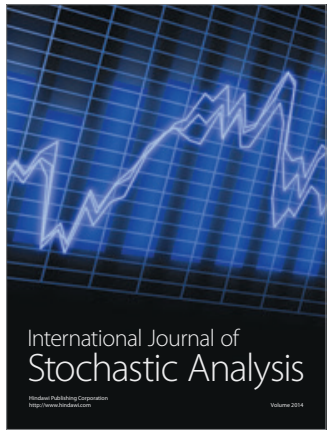

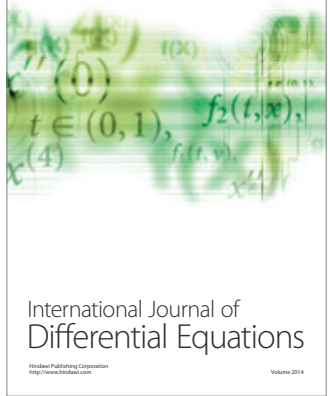
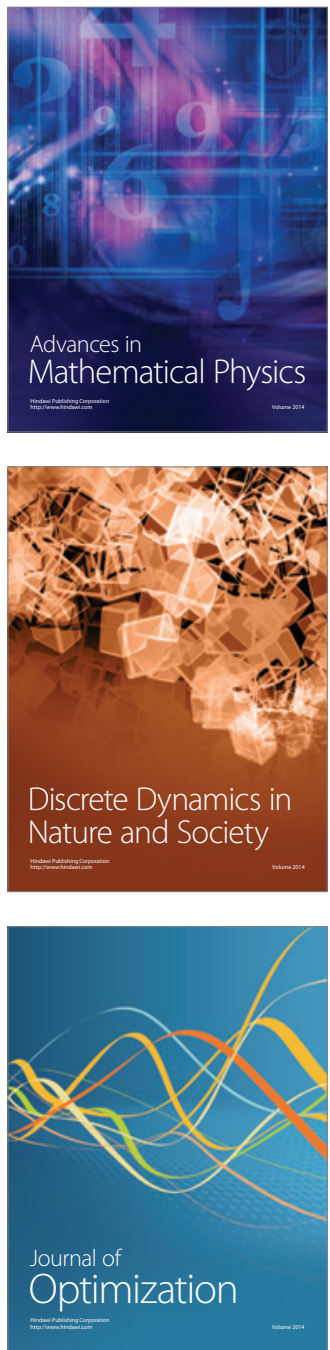